\title{
Finite groups in which some particular subgroups are TI-subgroups
}

\author{
Jiangtao Shi and Cui Zhang
}




\title{
FINITE GROUPS IN WHICH SOME PARTICULAR SUBGROUPS ARE TI-SUBGROUPS
}

\author{
JIANGTAO SHI AND CUI ZHANG
}

Received 6 June, 2013

\begin{abstract}
We prove that $G$ is a group in which all noncyclic subgroups are TI-subgroups if and only if all noncyclic subgroups of $G$ are normal in $G$. Moreover, we classify groups in which all subgroups of even order are TI-subgroups.
\end{abstract}

2010 Mathematics Subject Classification: 20D10

Keywords: noncyclic subgroup, subgroup of even order, TI-subgroup, normal

\section{INTRODUCTION}

All groups in this paper are considered to be finite. Let $G$ be a group and $M$ a subgroup of $G$. It is known that $M$ is called a TI-subgroup of $G$ if $M \cap M^{g}=1$ or $M$ for any $g \in G$.

In [5], Walls studied groups in which all subgroups are TI-subgroups. In [1], Guo, $\mathrm{Li}$ and Flavell classified groups in which all abelian subgroups are TI-subgroups. As a generalization of [1], we showed in [3] that $G$ is a group in which all nonabelian subgroups are TI-subgroups if and only if all nonabelian subgroups of $G$ are normal in $G$, and we showed in [4] that $G$ is a group in which all nonnilpotent subgroups are TI-subgroups if and only if all nonnilpotent subgroups of $G$ are normal in $G$.

In this paper, as a further generalization of above references, we first classify groups in which all noncyclic subgroups are TI-subgroups. We call such groups NCTIgroups. For NCTI-groups, we have the following result, the proof of which is given in Section 2.

Theorem 1. A group G is an NCTI-group if and only if all noncyclic subgroups of $G$ are normal in $G$.

Remark 1. It is easy to show that any NCTI-group is solvable but it might not be supersolvable. For example, the alternating group $A_{4}$ is an NCTI-group but $A_{4}$ is nonsupersolvable.

The first author was supported by NSFC, Grant No. 11201401 and 11361075 . 
We say that a group $G$ has nontrivial even order if $|G|$ is even but $|G| \neq 2$. In the following, we will classify groups in which all subgroups of even order are TIsubgroups. We call such groups EOTI-groups. For EOTI-groups, we have the following result, the proof of which is given in Section 3.

Theorem 2. A group $G$ is a EOTI-group if and only if one of the following statements holds:

(1) all subgroups of $G$ of nontrivial even order are normal in $G$.

(2) $G=Z_{p} \rtimes\langle g\rangle$ is a Frobenius group with kernel $Z_{p}$ and complement $\langle g\rangle$, where $p$ is an odd prime and $o(g)=2 n$ for some $n>1$.

Remark 2. We can easily get that any EOTI-group is solvable. But the alternating group $A_{4}$ shows that a EOTI-group might not be supersolvable.

Arguing as in proof of Theorem 2, we can obtain the following three results, which are generalizations of Theorem 1, [3] and [4] respectively. Here we omit their proofs.

Theorem 3. A group $G$ is a group in which all noncyclic subgroups of even order are TI-subgroups if and only if all noncyclic subgroups of $G$ of even order are normal in $G$.

Theorem 4. A group $G$ is a group in which all nonabelian subgroups of even order are TI-subgroups if and only if all nonabelian subgroups of $G$ of even order are normal in $G$.

Theorem 5. A group $G$ is a group in which all nonnilpotent subgroups of even order are TI-subgroups if and only if all nonnilpotent subgroups of $G$ of even order are normal in $G$.

\section{Proof of Theorem 1}

Proof. The sufficiency part is evident, we only need to prove the necessity part.

Let $G$ be an NCTI-group. Assume that $G$ has at least one nonnormal noncyclic subgroup. Choose $M$ as a nonnormal noncyclic subgroup of $G$ of largest order. Then, for any noncyclic subgroup $K$ of $G$, we have that $K$ is normal in $G$ if $M<K$.

We claim that $M=N_{G}(M)$. Otherwise, assume that $M<N_{G}(M)$. Let $L$ be a subgroup with $M<L \leq N_{G}(M)$ such that $M$ has prime index in $L$. By the choice of $M$, we have that $L$ is normal in $G$. Since $M$ is not normal in $G, N_{G}(M)<G$. Take $h \in G \backslash N_{G}(M)$. We have $M^{h}<L^{h}=L$. Since $M$ is a normal maximal subgroup of $L$ and $M^{h} \neq M$, we have $L=M M^{h}$. Note that $M$ is a TI-subgroup of $G$, which implies that $M \cap M^{h}=1$. Then $|L|=\left|M M^{h}\right|=|M|\left|M^{h}\right|$. It follows that $|M|=\left|M^{h}\right|=\frac{|L|}{|M|}=|L: M|$ is a prime, this contradicts that $M$ is noncyclic. Thus $M=N_{G}(M)$.

Then, by the hypothesis, $M \cap M^{a}=1$ for every $a \in G \backslash N_{G}(M)=G \backslash M$. We have that $G$ is a Frobenius group with $M$ being a Frobenius complement. Since $G$ 
is an NCTI-group, it follows that all nonabelian subgroups of $G$ are TI-groups. By [3], we have that all nonabelian subgroups of $G$ are normal in $G$. Then $M$ is abelian. It follows that every Sylow subgroup of $M$ must be cyclic by [2, Theorem 10.5.6]. This implies that $M$ is cyclic, a contradiction. Thus $G$ has no nonnormal noncyclic subgroup.

\section{Proof of Theorem 2}

Proof. (1) We first prove the necessity part. Let $G$ be a EOTI-group. Assume that $G$ has at least one nonnormal subgroup of nontrivial even order. Let $Q$ be any nonnormal subgroup of $G$ of nontrivial even order. Choose $L$ as a nonnormal subgroup of $G$ of nontrivial even order of largest order such that $Q \leq L$.

We claim that $L=N_{G}(L)$. Assume that $L<N_{G}(L)$. Let $K$ be a subgroup with $L<K \leq N_{G}(L)$ such that $L$ has prime index in $K$. By the choice of $L$, we have that $K$ is normal in $G$. Let $y$ be an element of $G \backslash N_{G}(L)$. Then $L \cap L^{y}=1$ by the hypothesis. It follows that $K=L L^{y}$ since $L$ is a normal maximal subgroup of $K$. Then $|L|=\left|L^{y}\right|=|K: L|$ is a prime, a contradiction. Thus $L=N_{G}(L)$.

It follows that $G$ is a Frobenius group with complement $L$. Let $N$ be the Frobenius kernel of $G$. We have $G=N \rtimes L$.

We claim that $L$ is a maximal subgroup of $G$. If $L$ is not a maximal subgroup of $G$. Let $M$ be a maximal subgroup of $G$ such that $L<M$. It is obvious that $M$ also has nontrivial even order. By the choice of $L$, we have that $M$ is normal in $G$. Since $G$ is a Frobenius group, it follows that either $M \leq N$ or $N<M$. If $M \leq N$, then $L<N$, a contradiction. If $N<M$, then $G=N \rtimes L \leq M$, again a contradiction. Thus $L$ is a maximal subgroup of $G$.

It follows that $N$ is a minimal normal subgroup of $G$. Since $N$ is nilpotent by [2, Theorem 10.5.6], we can assume that $N=Z_{p}{ }^{m}$, where $m \geq 1$ is a positive integer. Let $d$ be an element of $L$ of order 2. Since $G$ is a Frobenius group and $N$ is abelian, we have $e^{d}=e^{-1}$ for every $1 \neq e \in N$. Thus $\langle e\rangle \rtimes\langle d\rangle$ is a subgroup of $G$.

We claim that $\langle e\rangle \rtimes\langle d\rangle$ is normal in $G$. Note that $|\langle e\rangle \rtimes\langle d\rangle|=2 p$, where $p$ is an odd prime. If $\langle e\rangle \rtimes\langle d\rangle$ is not normal in $G$. Arguing as the subgroup $Q$, there exists a nonnormal subgroup $T$ of $G$ of nontrivial even order of largest order such that $\langle e\rangle \rtimes\langle d\rangle \leq T$. And we have that $T$ is also a Frobnius complement of $G$. But $N \cap T \geq\langle e\rangle \neq 1$, a contradiction. Thus $\langle e\rangle \rtimes\langle d\rangle$ is normal in $G$.

Since $\langle e\rangle \rtimes\langle d\rangle \not \leq N=Z_{p}{ }^{m}$, we have $Z_{p}{ }^{m}<\langle e\rangle \rtimes\langle d\rangle$. It follows that $m=1$. Thus $N=Z_{p}$ is cyclic. By the N/C-theorem, since $C_{G}(N)=N$ it follows that $L$ is isomorphic to a subgroup of $\operatorname{Aut}\left(Z_{p}\right) \cong Z_{p-1}$. Then $L$ is a cyclic group of order $2 n$ for some $n>1$. Assume that $L=\langle g\rangle$. We have $G=Z_{p} \rtimes\langle g\rangle$, where $p$ is an odd prime and $o(g)=2 n$ for some $n>1$.

(2) Now we prove the sufficiency part. 
(i) If all subgroups of $G$ of nontrivial even order are normal in $G$, then $G$ is obviously a EOTI-group.

(ii) Assume that $G=Z_{p} \rtimes\langle g\rangle$ is a Frobenius group with kernel $Z_{p}$ and complement $\langle g\rangle$, where $p$ is an odd prime and $o(g)=2 n$ for some $n>1$. Let $S$ be any subgroup of $G$ of even order. It is obvious that $S \cap Z_{p}=1$ or $Z_{p}$.

If $S \cap Z_{p}=1$, we have $S \leq\langle g\rangle^{f}$ for some $f \in G$. Obviously $S$ is not normal in $G$. Then $N_{G}(S)=\langle g\rangle^{f}$. We have $S \cap S^{x} \leq\langle g\rangle^{f} \cap\left(\langle g\rangle^{f}\right)^{x}=1$ for every $x \in G \backslash\langle g\rangle^{f}=G \backslash N_{G}(S)$.

If $S \cap Z_{p}=Z_{p}$, then $Z_{p}<S$. We have $S=S \cap G=S \cap\left(Z_{p} \rtimes\langle g\rangle\right)=Z_{p} \rtimes$ $(S \cap\langle g\rangle)$. Then $S$ is normal in $G$.

It follows that $S$ is always a TI-subgroup of $G$ whenever $S \cap Z_{p}=1$ or $Z_{p}$. Then $G$ is also a EOTI-group.

\section{ACKNOWLEDGEMENT}

The authors are grateful to the referee who gives valuable comments and suggestions.

\section{REFERENCES}

[1] X. Guo, S. Li, and P. Flavell, "Finite groups whose Abelian subgroups are TI-subgroups," J. Algebra, vol. 307, no. 2, pp. 565-569, 2007.

[2] D. J. Robinson, A course in the theory of groups, 2nd ed., ser. Graduate Texts in Mathematics. New York: Springer-Verlag, 1995, vol. 80.

[3] J. Shi and C. Zhang, "Finite groups in which all nonabelian subgroups are TI-subgroups," J. Algebra Appl., vol. 13, 1350074, 2014.

[4] J. Shi and C. Zhang, "A note on TI-subgroups of a finite group," Algebra Colloq., to appear.

[5] G. Walls, "Trivial intersection groups," Arch. Math., vol. 32, pp. 1-4, 1979.

Authors' addresses

\section{Jiangtao Shi}

Yantai University, School of Mathematics and Information Science, 264005 Yantai, China

E-mail address: shijt@math.pku.edu.cn

\section{Cui Zhang}

Technical University of Denmark, Department of Applied Mathematics and Computer Science, DK2800 Lyngby, Denmark

E-mail address: cuizhang2008@gmail.com 